\title{
Holistic Blockchain Approach to Foster Trust, Privacy and Security in IoT based Ambient Assisted Living
}

\author{
Akpanakak Mkpa \\ Department of Computing and \\ Technology \\ Anglia Ruskin University \\ Cambridge. UK \\ akpanakak.mkpa@pgr.anglia.ac.uk
}

\author{
Jeannette Chin \\ Department of Computing and \\ Technology \\ Anglia Ruskin University \\ Cambridge. UK
}

\author{
Andrian Winkles \\ Department of Computing and \\ Technology \\ Anglia Ruskin University \\ Cambridge. UK
}

\begin{abstract}
The application of blockchains techniques in the Internet of Things (IoT) is gaining much attention with new solutions proposed in diverse areas of the IoT. Conventionally IoT systems are designed to follow the centralized paradigm where security and privacy control is vested on a "trusted" third-party. This design leaves the user at the mercy of a sovereign broker and in addition, susceptible to several attacks. This implicit trust and the inferred reliability of centralized systems have been challenged recently following several privacy violations and personal data breaches. Consequently, there is a call for more secure decentralized systems that allows for finer control of user privacy while providing secure communication. Propitiously, the blockchain holds much promise and may provide the necessary framework for the design of a secure IoT system that guarantees fine-grained user privacy in a trustless manner. In this paper, we propose a holistic blockchain-based decentralised model for Assisted Ambient Living (AAL) environment. The nodes in our proposed model utilise smart contracts to define interaction rules while working collaboratively to contribute storage and computing resources. Based on blockchain technique our proposed model offers trustless interactions and enhanced user's privacy. Integrating with Interplanetary File System (IPFS) for distributed storage, the proposed model addresses the shortfall of storage constraints exhibit in many IoT systems.
\end{abstract}

Keywords-blockchain; Internet of Things; AAL; Trust and Privacy; Security; Smart Contracts; Ethereum Swarm, IPFS

\section{INTRODUCTION}

The internet of things is described as potentially amongst the most significant disruptive technologies of the $21 \mathrm{st}$ century, and it believed to be the angular stone of the information and Communication Technology (ICT) market in the coming years [1]. In 2016, there were approximately 13.3 Million Internet of Things (IoT) connections in the UK and this is expected to grow at a compound annual growth rate (CAGR) of approximately $36 \%$ to 155.7 million connections at the end of 2024 [2]. Similarly, Cisco forecasts 50 billion devices will be connected worldwide by 2020 , with an average of about 16 IoT devices per person; a potential market in excess of \$14 trillion [3]. A recent study shows that by 2025 , the IoT will form an integral part of everyday things such as household, furniture, wearable health systems, food packaging, clothing and paper documents [4].

IoT allows the integration of tiny pervasive devices into our daily lives. This, in turn, enables the digital world to directly affect our physical space through sensing and automation. While this integration presents several opportunities for improved services, it also exposes us to threats and attacks that prevail in the digital space [5]. By way of an example is Ambient Assisted Living (AAL) environment - a technical system built to support the elderly and infirmed to improving their safety and enhancing dayto-day living [6]. IoT in AAL environment utilizes intelligent devices in the homes of the elderly to continuously monitor and collect information, both system and user data, and forward them to a centralized system for processing and analysis. The data gathered reveals patterns that can be used by healthcare professionals to assist with diagnosis or collaborative care or treatment of ailments [7]. It has been suggested that IoT plays a significant role in AAL for improving wellbeing, safety and healthcare of millions of elderly people worldwide due to the nature of its power of connectivity and sensing. Hence vital health statistics can be provided by constantly gathering data from the body environment, which in turn helps longevity [9].

In this paper, we first present security and privacy challenges of AAL, explore existing implementations of AAL and discuss the drawbacks of these implementations. We then propose a holistic approach to address the drawbacks. We argue the use of blockchain, smart contracts and IPFS will enable trustless transactions in the distributed AAL environment enhancing user's privacy and security. The rest of the paper is organized as follows: Section I and II exploring the background information on IoT and AAL and identifying its opportunities and challenges. In Section III, we introduce the use of blockchain as a possible solution to the challenges of the Internet of Things in Ambient Assisted Living environment and also investigate the pitfalls of using blockchain in AAL. To conclude this section introduces our proposed holistic model to address these problems. In Section IV we conclude the paper and set up our future work.

\section{INTERNET OF THINGS AND AMBIENT ASSISTED LIVING}

\section{A. Background and Context}

Ambient assisted living is gaining significant attention as a result of the fast-growing demographic of the ageing population [10]. This put a great burden on the traditional care infrastructure, thereby challenges the viability of conventional elderly care systems. According to [36] successful utilization of AAL in association with IoT technologies promises to greatly lower operational cost, facilitate collaborative care, and encourage the elderly to 
live independently. However, Security and user privacy protection remain a major challenge [11] to IoT-AAL integration (Fig 1). Information collected by IoT devices used therein are of interest to many players and present an attractive target to cybercriminals. For instance, user personal information can be sold to third parties where it will be analyzed to reveal patterns that might affect the user's chances of fair treatment, especially in health insurance. Users are also worried about their critical health data being tampered with or stored in untrusted servers, as disclosure or abuse of personal information can lead to property damage. This is as evidenced in the recent Edward Snowden and Cambridge Analytica Saga [13], which caused great mistrust in centralized processing of personal information, leading to an intensified call for fine-grained control of user privacy. This abuse of trust has led to a drastic decline in the adoption of IoT technologies in recent times, especially in home automation and AAL [2]. Consequently, research in AAL has intensified and many systems, methods and prototypes have been developed to provide solutions to security and privacy concerns. Minetti et al [14] presented smart hospitals system (SHS); an IoTaware system which provides automatic patient and assets tracking in hospitals and care homes. Suntiamorntut et al [15] proposed an affordable system for private homes to assist the elderly to live independently. Other approaches such as Bodyguarding Heart [16] rely on wearable devices in combination with smartphones to monitor vital signs and other health information. Wellness [17] incorporated sensors to develop home automation systems where alerts are sent to family members or caregivers in events of an emergency. The European Union is funding large scale projects such as GiraffPlus and InCassa, to assist with AAL implementation. GiraffPlus [18] employed physiological and environmental sensors placed in the home of the elderly which captures data to be subsequently analyzed by intelligent systems. It also incorporated a telepresence robot named Giraffe that can be controlled remotely to assist the elder or serve a social role [19].

Although implementations cited above provide a level of private using access control mechanism, users are still exposed to internal abuse or misuse of privacy information collected and managed by the broker. To this end, systems built upon the centralized brokered model will fail to guarantee the advocated level of privacy control and user autonomy that is required of future IoT and AAL implementation. The brokered model leaves the user at the mercy of a sovereign third-party and in addition, susceptible to several attacks. As a result, implicit trust and the reliability of centralized systems has been recently challenged [22], therefore calling for more secure decentralized systems that allow for fine-grained control of user privacy. Consequently, the trust framework of the existing Internet and IoT, which requires the user to inherently trust the broker, needs to be reviewed to meet the requirements of future IoT [23]. IoT is an entirely new paradigm presenting with lots of new challenges require a holistic approach from product development to the needs of analysis and associated business models to support such products. Consequently, the potential benefits of IoT/AAL cannot be fully utilized except new methodologies, approaches and techniques are developed to meet IoT requirements in terms of trust, privacy and security [24].

\section{B. Opportunities and Challenges}

Home automation is currently the slowest area of IoT penetration because consumers have failed to embrace the potentials for fear of privacy invasion [25]. To this end, current solutions based on the brokered model and "security through obscurity" approach fail to meet this persistent user demands. However, models that employ "security through transparency" hold potential [25] for future IoT development will favor a scenario where users have fine-grained control over access to sensitive data collected and benefit from proceeds of analysis or the use of their personal data. This calls for a shift in the conventional approach to security in IoT to embrace new paradigms that radically lower the cost, allowing users to extract value for data exchange, promoting privacy and autonomy, while also providing adequate security. Crucially, it implies different challenges in particular for all implementation utilizing the clientserver/brokered model cannot effectively satisfy the call for fine-grained control of user privacy and trust requirement of the new paradigm because the broker will always have sovereign control and access to user information hence, fostering the possibility of misuse and abuse. We argue that new models built upon decentralized paradigm hold much promise. Such implementation must permit secure communication amongst peers, allowing trustworthy usercontrolled interactions, development of micro-services, user autonomy and transparency at all times

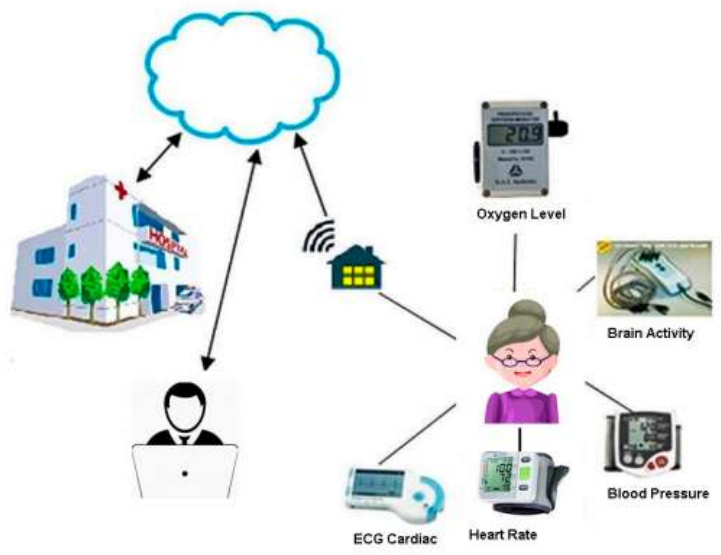

Fig. 1. A simplified representation of current implementation of IoT in $A A L$.

\section{BLOCKCHAIN}

A paper published by Satoshi Nakamoto in 2008 introduced the concept of Blockchain to enable entities to transact in a safe and secure manner without the need for a trusted third party [26]. Blockchain, originally designed for use in the financial sector, has grown in recent years and has been adapted to provide solutions in different sectors of the economy such as energy [27], finance, intelligent transport systems legal, IoT, and healthcare [28].

Blockchain represents a new approach to service delivery and start-ups are seeking new ways to incorporate its abilities to enable transactions between unreliable actors to be transparent, highly resistant and auditable. Put simply; blockchain can be viewed as a database that maintains a continuously growing set of time-stamped transaction records where each hashed block of transactions is chained and linked to the hash of the previous one [29] [30]. 
Blockchain permits multiple nodes on a network to transact securely without relying on a trusted third party. Contrary to the brokered model which mandates users to trust the broker implicitly, a blockchain model relies on cryptographic proofs rather than trust [31], thereby enabling disparate parties to transact securely without the need for a trusted third party. This forms the basis of its efficiency in developing decentralized trustless systems.

Despite the promise of blockchain to IoT and AAL, storage capacity remains a major challenge [32] in practice owing to the fact that Blockchain was originally designed to append tiny records of financial transactions to a ledger and lacks the capacity to store large data streams generated by pervasive devices used in IoT and AAL. The ledger grows continually as more blocks are created, putting more pressure on the resources, hence reducing its ability to scale considerably [33].

\section{A. A collaborative holistic approach to AAL using Blockchain, Smart Contracts, IPFS decentralised storage and Ethereum Swarm as a possible solution}

We propose a collaborative holistic approach to address the storage problem in blockchain-based AAL environment using decentralized storage - the InterPlanetary File System (IPFS) [34] [41], together with Smart Contracts and Ethereum Swarm (Fig 2). Blockchain has been explained in the previous section, we will describe the remaining components individually:

IPFS is a $\mathrm{p} 2 \mathrm{p}$ hypermedia protocol that combines the distributed hash table, an incentivized block exchange and self-certifying namespace to coordinate a network of untrusted peers to cooperate in distributing files to each other [35]. Built in line the technology behind bit torrent, IPFS synthesizes the best ideas in distributed file systems built to date to connect all computing devices with the same system of files [34]. IPFS provides a high throughput contentaddressed block storage model that exhibits no single point of failure and best suited in an environment where nodes do not need to trust each other [34]. In addition, IPFS can handle big data with ease. It is well suited for hosting and distributing petabyte dataset, high-volume high definition on-demand and real-time media streaming, computing on large data across organizations, versioning and linking of massive data sets and preventing accidental disappearance of important files.

Similarly, Swarm is an Ethereum decentralized content distribution service and storage platform. It is used for storing Ethereum public records and Distributing Applications (Dapps) code. Ethereum Swarm is censorship resistant, like blockchain, it has no single point of failure and supports a built-in incentive mechanism for peers that pool contribute their storage and bandwidth. It is maintained by participating peers who contributes their storage and bandwidth resources to facilitate content distribution. Swarm is designed with a mechanism to ensure the availability of unpopular contents and scales easily.

Smart Contracts are a set of executable functions and state variables that govern the interaction of nodes in a blockchain network. Smart contracts reside in the blockchain and are executed when transactions are addressed or sent to it. Smart contracts define input parameters that must be supplied by the interacting calls, which is used to manipulate the state of the contract based on the publicly available logic contained within the contract. Once compiled, smart contracts are uploaded to the blockchain which assigns a unique address to each smart contract. smart contracts can operate autonomously interacting with other smart contracts or devices in the blockchain.

In our proposed holistic model mentioned above, an IoTbased AAL environment will consist of a network of interacting sensors such as motion sensors, fall detection sensors, environmental sensors, wearable devices, and smart appliances. These sensors and smart appliances will collaborate to provide relevant data necessary for effective health monitoring of the occupant. Using a machine learning technique, the data collected will be intelligently analyzed for a situation that may trigger a response or intervention by the healthcare team. Such triggers or any other relevant data will be pushed into the blockchain-IPFS network for storage. In cases where the supplied data is large, the hash of the data will be stored in the blockchain, while the bulk data is stored in the distributed storage network of nodes that contribute storage facility for a reward (IPFS, Swarm etc.) Content addressing will be used to properly index the files for access when needed. To access information stored in the blockchain and the distributed storage network, smart contracts will be employed to ensure a user-controlled access to data. The smart contract will define which data is accessed by whom, based on the role and agreement with the data owner. This will also allow the user to trade data for value, thereby extracting some form of financial or concessional benefits from services rendered by companies using data generated by these devices. Furthermore, any information of importance can be stored in the blockchain as defined by the user and other key people in the IoT-AAL ecosystem.

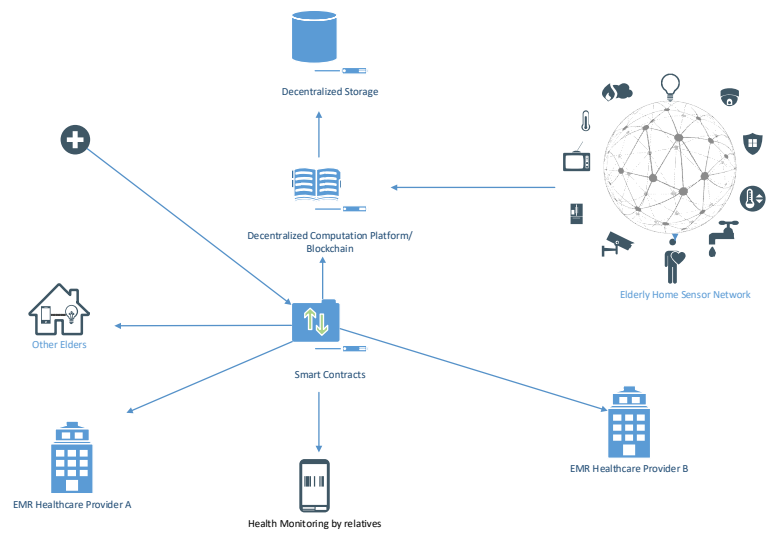

Fig. 2. Diagrammatic representation of proposed holistic model and system architecture

\section{CONCLUSION AND FUTURE WORK}

Although IoT promises great cost reduction and enhancement to AAL and elderly care, there are many barriers that need to be overcome in order to gain user trust and improve the technology adoption rate. We propose a holistic model to address this problem. With the use of blockchain technique, smart contract, IPFS and Swarm, our proposed holistic model enhances privacy and security by (1) using smart contracts which define rules for interaction with users and data as a mean to empower user's control and (2) offering trustless transactions between nodes on the network. The storage constraints associated with blockchain in IoT is resolved by leveraging distributed scalable IPFS platform. 
The future work is to implement this proposed holistic model and conduct critical system and user evaluations for futureproof. We aimed to use resource-constrained devices in our testing environment. Our first target users are elderly homes in the UK. Modifications will be made where necessary to adapt some associated platforms to meet the needs of AAL.

\section{REFERENCES}

11] S. Sicari, A. Rizzardi, L. A. Grieco, and A. Coen-Porisini, "Security, privacy and trust in Internet of Things: The road ahead," Comput. Networks, 2015.

[2] T. Winchcomb, S. Massey, and P. Beastall, "Review of latest developments in the Internet of Things," vol. 1636, no. 1636, pp. 1-143, 2017.

[3] Taylor Stuart, "10 Predictions for the Future of the Internet of Things," $2015 . \quad$ [Online]. Available: https://blogs.cisco.com/cle/10-predictions-for-the-future-of-theinternet-of-things. [Accessed: 15-Jan-2019].

[4] X. Caron, R. Bosua, S. B. Maynard, and A. Ahmad, "The Internet of Things (IoT) and its impact on individual privacy: An Australian perspective," Comput. Law Secur. Rev, 2016.

[5] C. Pahl, N. EL Ioini, and S. Helmer, "A Decision Framework for Blockchain Platforms for IoT and Edge Computing," Proc. 3rd Int. Conf. Internet Things, Big Data Secur., no. March, pp. 105$113,2018$.

[6] A. Dohr, R. Modre-Osprian, M. Drobics, D. Hayn, and G. Schreier, "The internet of things for ambient assisted living," ITNG2010 - 7th Int. Conf. Inf. Technol. New Gener, pp. 804-809, 2010 .

[7] J. Ko, C. Lu, M. B. Srivastava, J. A. Stankovic, A. Terzis, and M. Welsh, "Wireless sensor networks for healthcare," in Proceedings of the IEEE, 2010, vol. 98, no. 11, pp. 1947-1960.

[9] D. Metcalf, S. T. J. Milliard, M. Gomez, and M. Schwartz, "Wearables and the internet of things for health: Wearable, interconnected devices promise more efficient and comprehensive health care," IEEE Pulse, vol. 7, no. 5, pp. 3539,2016

[10] P. Rothenpieler, C. Becker, and S. Fischer, "Privacy concerns in a remote monitoring and social networking platform for assisted living," 2011

[11] M. Memon, S. R. Wagner, C. F. Pedersen, F. H. Aysha Beevi, and F. O. Hansen, "Ambient Assisted Living healthcare frameworks, platforms, standards, and quality attributes," Sensors (Switzerland), vol. 14, no. 3, pp. 4312-4341, 2014

[12] J. Qi, P. Yang, G. Min, O. Amft, F. Dong, and L. Xu, "Advanced internet of things for personalised healthcare systems: A survey," Pervasive Mob. Comput., vol. 41, pp. 132-149, 2017.

[13] S. Landau and | Google, "Highlights from Making Sense of Snowden, Part II: What's Significant in the NSA Revelations."

[14] L. Catarinucci et al., "An IoT-Aware Architecture for Smart Healthcare Systems," IEEE Internet Things J., vol. 2, no. 6, pp. $515-526,2015$.

[15] W. Suntiamorntut, S. Charoenpanyasak, and J. Ruksachum, "An elderly assisted living system with wireless sensor networks," Proc. 2011 4th Jt. IFIP Wirel. Mob. Netw. Conf. WMNC 2011, pp. $1-6,2011$.

[16] Preventice Solutions, "Preventice - BodyGuardian Heart," 2015. [Online]. Available: https://www.preventicesolutions.com/services/body-guardianheart.html. [Accessed: 23-Jan-2019].

[17] Alarm Systems, "Wellness: Secure Independent Living System from Alarm.com." [Online]. Available: https://www.alarm.com/productservices/wellnessproviders.aspx. [Accessed: 23-Jan-2019].

[18] "Giraff Plus." [Online]. Available: http://www.giraffplus.eu/ [Accessed: 23-Jan-2019].

[19] A. Cesta et al., "Steps Toward End-to-End Personalized AAL Services," 2013.

[20] B. Bhargava, C. Farkas, L. Lilien, and F. Makedon, "Trust, Privacy, and Security," 2003.

[21] M. Darwish, E. Senn, C. Lohr, and Y. Kermarrec, "A comparison between ambient assisted living systems," Lect. Notes Comput. Sci. (including Subser. Lect. Notes Artif. Intell. Lect. Notes Bioinformatics), vol. 8456, pp. 231-237, 2015.

[22] W. Sun, Z. Cai, Y. Li, F. Liu, S. Fang, and G. Wang, "Security and Privacy in the Medical Internet of Things: A Review," Secur Commun. Networks, vol. 2018, 2018.

[23] T. M. Fernández-Caramés and P. Fraga-Lamas, "A Review on the Use of Blockchain for the Internet of Things," IEEE Access, vol. 6, pp. 32979-33001, 2018.

[24] M. Abomhara, "Security and Privacy in the Internet of Things : Current Status and Open Issues," Priv. Secur. Mob. Syst. (PRISMS), 2014 Int. Conf., pp. 1-8, 2014.

[25] P. Brody and V. Pureswaran, "Device democracy: Saving the future of the Internet of Things IBM," IBM Glob. Bus. Serv. Exec. Rep., 2015.

[26] S. Nakamoto, "Bitcoin: A Peer-to-Peer Electronic Cash System," 2008.

[27] N. Z. Aitzhan and D. Svetinovic, "Security and Privacy in Decentralized Energy Trading Through Multi-Signatures, Blockchain and Anonymous Messaging Streams," IEEE Trans. Dependable Secur. Comput., vol. 15, no. 5, pp. 840-852, 2018

[28] A. Azaria, A. Ekblaw, T. Vieira, and A. Lippman, "MedRec: Using blockchain for medical data access and permission management," Proc. - 2016 2nd Int. Conf. Open Big Data, OBD 2016, pp. 25-30, 2016

[29] A. M. Antonopoulos, Mastering Bitcoin, vol. 50, no. 4. 2014.

[30] M. Abomhara and G. M. Køien, "Cyber Security and the Internet of Things: Vulnerabilities, Threats, Intruders and Attacks,” 2015.

[31] G. Zyskind, O. Nathan, and A. S. Pentland, "Decentralizing privacy: Using blockchain to protect personal data," in Proceedings - 2015 IEEE Security and Privacy Workshops, SPW 2015,2015 .

[32] A. Reyna, C. Martín, J. Chen, E. Soler, and M. Díaz, "On blockchain and its integration with IoT. Challenges and opportunities," Futur. Gener. Comput. Syst., vol. 88, no. 2018, pp. 173-190, 2018.

[33] B. Arshdeep and M. Vijay, Blockchain Applications. 2017.

[34] J. Benet, "IPFS - Content Addressed, Versioned, P2P File System," 2017.

[35] Y. Chen, H. Li, K. Li, and J. Zhang, "An improved P2P file system scheme based on IPSF and Blockchain," in 2017 IEEE International Conference on Big data, 2017.

[36] R. Fernandez Horcajada et al., "Final Report. A Study concerning a Market Observatory in the Ambient Assisted Living field," 2014

[37] R. Fernandez Horcajada et al., "Final Report. A Study concerning a Market Observatory in the Ambient Assisted Living field," 2014

[38] J Chin; V Callaghan; S. B. Allouch; "The Internet-of-Things: Reflections on the past, present and future from a user-centered 